\title{
Confiabilidad y validez de un instrumento para medir la inteligencia emocional en estudiantes de posgrado
}

\section{Reliability and validity of an instrument to measure emotional intelligence in graduate students}

MOSCOSO-PACHECO, Johanna*†, SANDOVAL-CARAVEO, María del Carmen, AGUILARMORALES, Norma

Universidad Juárez Autónoma de Tabasco

ID $1^{\mathrm{er}}$ Autor: Johanna, Moscoso-Pacheco / ORC ID: 0000-0002-9112-7407, Researcher ID Thomson: O-2676-2017, CVU CONACYT ID: 824555

ID $1^{\text {er }}$ Coautor: María del Carmen, Sandoval-Caraveo / ORC ID: 0000-0002-5482-3032, Researcher ID Thomson: K-93212017, CVU CONACYT ID: 515768

ID $2^{\text {do }}$ Coautor: Norma, Aguilar- Morales / ORC ID: 0000-0002-7292-3029, Researcher ID Thomson: K-8796-2018, CVU CONACYT ID: 478554

DOI: $10.35429 /$ JPDL.2019.16.5.28.34

Recibido 29 de Junio, 2019; Aceptado 30 Septiembre, 2019

Resumen

Objetivo: Calcular la confiabilidad y validez del instrumento Genos de Inteligencia Emocional versión concisa, como un modelo de instrumento de autoevaluación confiable para usos de investigación aplicado en estudiantes de posgrado de una universidad pública del sureste de México. Metodología: La investigación tiene un enfoque cuantitativo, con un diseño no experimental transversal, participaron 332 estudiantes de maestría y doctorado. La confiabilidad del instrumento se calculó a través del coeficiente Alpha de Cronbach $\alpha=$ 0.93. Para la validez se realizó un análisis factorial exploratorio con el método de máxima verosimilitud con rotación de Oblimin directo, la adecuación de la muestra de KMO fue de 0.927 y la prueba de esfericidad de Barlett fue significativa $(\mathrm{p}=.000)$ que explicaron el $53.89 \%$ de la varianza. La factorización del instrumento fusionó dos de sus dimensiones y se obtuvo un instrumento con igual cantidad de ítems en seis dimensiones Contribución: Los resultados permitieron confirmar la fiabilidad y validez del instrumento Genos de Inteligencia Emocional lo que contribuye a contar con un instrumento que se suma a otras herramientas existentes para autoevaluar la inteligencia emocional.

Confiabilidad, Inteligencia emocional, Genos IE

\begin{abstract}
Objective: Calculate the reliability and validity of the Genos Emotional Intelligence instrument concise version, as a reliable self-assessment instrument model for applied research applications in postgraduate students of a public university in southeastern Mexico. Methodology: The research has a quantitative approach, with a nonexperimental cross-sectional design, 332 master's and doctoral students participated. The reliability of the instrument was calculated through the Cronbach's alpha coefficient $\alpha=0.93$. For the validity an exploratory factor analysis was performed with the maximum likelihood method with direct Oblimin rotation, the adequacy of the KMO sample was 0.927 and the Barlett sphericity test was significant $(\mathrm{p}=.000)$ that explained $53.89 \%$ of the variance. The factorization of the instrument merged two of its dimensions and an instrument with the same number of items in six dimensions was obtained. Contribution: The results allowed confirming the reliability and validity of the Genos Emotional Intelligence instrument, which contributes to an instrument that adds to other existing tools to self-assess emotional intelligence.
\end{abstract}

Reliability, Emotional intelligence, Genos IE

Citación: MOSCOSO-PACHECO, Johanna, SANDOVAL-CARAVEO, María del Carmen, AGUILAR- MORALES, Norma. Confiabilidad y validez de un instrumento para medir la inteligencia emocional en estudiantes de posgrado. Revista de Filosofía y Cotidianidad. 2019, 5-16: 28-34

\footnotetext{
* Correspondencia del Autor (ymospach@gmail.com)

$\dagger$ Investigador contribuyendo como primer Autor.
} 


\section{Introducción}

Cuando las empresas realicen un proceso de contratación, buscarán no solo satisfacer la frase "el hombre adecuado para el puesto adecuado" sino también seres humanos con las competencias requeridas para el puesto en cuestión y el valor agregado que da la inteligencia emocional a las personas. $\mathrm{La}$ realidad hoy es que en las organizaciones existen individuos que poseen grandes habilidades administrativas y organizacionales, capaces de tomar decisiones basadas en su gran experiencia, pero carentes de un liderazgo que logre mover a los colaboradores a sentirse parte de la organización y sobre todo a generar un compromiso que les permita comprometerse con el trabajo y el logro; cuando un trabajador está motivado y su líder es empático a las diversas situaciones humanas que viven, se logra un equipo de trabajo con sinergia logrando una organización emocionalmente inteligente.

El objetivo de este trabajo fue calcular la confiabilidad y validez del instrumento Genos de Inteligencia Emocional (IE) en su versión concisa, fue aplicado a estudiantes de posgrado de una universidad pública con el fin de contribuir a las ciencias administrativas con una herramienta aceptable para su aplicación y contribuir con ello a las competencias de los nuevos líderes, no se duda de las capacidades académicas, puesto que un nivel de posgrado requiere de un compromiso consigo mismo, así como de un deseo de trascender más allá del estudio básico.

\section{Inteligencia Emocional}

La emoción es la reacción como respuesta a un estímulo, el cual se experimenta de manera individual (Calderón, Gonzaléz, Salazar, \& Washburn, 2012), la inteligencia emocional considera las habilidades de percepción, valoración, expresión e interacción de las emociones (Fernández-Berrocal, Extremera y Durán, 2003; Extremera y Fernandez, 2005; Trujillo y Rivas, 2005; García-Izquierdo, García-Izquierdo y Ramos-Villagrasa, 2007; Núñez et al., 2008; Zárate y Matviuk, 2012; Pino y Aguilar, 2013; De José, Danvila, MartínezLópez, y Sastre, 2013; Hernández-Vargas y Dickinson-Bannack, 2014; Duque, Garcia, y Hurtado, 2017; Newman, Purse, Smith, y Broderick, 2017).
Dicho concepto fue dado a conocer por Peter Salovey y Johnn Mayer (Goleman, 2015) no solo en el ámbito de las relaciones familiares, sino con todos aquellos con quienes tratamos, ya sean conocidos, clientes, compañeros de trabajo, es decir con cualquier individuo donde se tenga alguna relación social, ahí está presente la inteligencia emocional (OCDE, 2005).

El concepto trasciende de la psicología y es llevado a los ámbitos de las organizaciones, desarrollando capacidades que aporten a la creación de organizaciones más inteligentes (Lozano y González, 2015; Agreda, 2016; Serrano, 2017) identificándose a través de una serie de constructos administrativos e influyendo en ciertos procesos de la organización como son, la selección del personal, el liderazgo, la satisfacción laboral, la solución de conflictos entre otros.

Las emociones son una parte del ser humano, que influye en sus estados de ánimo, es decir impactan en el comportamiento de los individuos en algún momento y situación determinada, por tal razón es de importancia estudiar cómo impactan las emociones en las habilidades y capacidades de cada persona y los resultados en su desempeño (Duque, García, y Hurtado, 2017).

Al considerar a un trabajador para un puesto determinado, es de vital importancia las competencias adecuadas al perfil del puesto, particularidades que la organización considera relevantes, así como el conjunto de actitudes, valores, habilidades y rasgos que forman su personalidad, por tal situación es indispensable considerar alternativas que ofrezcan un mayor conocimiento del individuo (Gil, 2007).

\section{Instrumentos aplicables a la inteligencia emocional}

Existen diversos instrumentos que permiten la autoevaluación de la inteligencia emocional, los cuales están enfocados a la personalidad del individuo y orientados a lo que se busca de ellos, apoyar en el desarrollo de sus habilidades.

En la tabla 1 se mencionan las características de tres modelos de instrumentos de inteligencia emocional con sus dimensiones y número de ítems, los cuales han sido utilizados en diversos ámbitos. 


\begin{tabular}{|c|c|}
\hline Modelo & Características \\
\hline $\begin{array}{lr}\text { MSCEIS } & \text { (Mayer, } \\
\text { Salovey, Caruso, } \\
\text { Emotional Intelligence } \\
\text { Test) Modelo de } \\
\text { Inteligencia Emocional } \\
\text { de Mayer y Salovey. }\end{array}$ & $\begin{array}{l}\text { Modelo compuesto por cuatro } \\
\text { dimensiones con } 141 \text { ítems. } \\
\text { a) Percepción de emociones. } \\
\text { b) Emociones que faciliten el } \\
\text { pensamiento. } \\
\text { c) Comprensión emocional. } \\
\text { d) Manejo de emociones. }\end{array}$ \\
\hline $\begin{array}{lr}\text { El TMMS } & \text { (Trait } \\
\text { Mood } & \text { Meta- } \\
\text { desarrollado } & \text { Scale) } \\
\text { Salovey y } & \text { Mayer, para } \\
\text { evaluar } & \text { por } \\
\text { interpersonales } & \text { y } \\
\text { aspectos } & \text { emocionales } \\
\text { generales. } & \end{array}$ & $\begin{array}{l}\text { Consta de } 48 \text { ítems en su versión } \\
\text { "larga", con tres subescalas: } \\
21 \text { ítems: Conocimiento de las } \\
\text { Emociones. } \\
15 \text { ítems: claridad con respecto a } \\
\text { las emociones. } \\
12 \text { ítems: regulación emocional. } \\
\text { Enfocada a estudiantes. }\end{array}$ \\
\hline 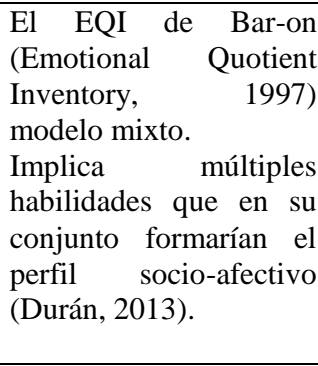 & $\begin{array}{l}\text { Lo forma } 133 \text { Ítems distribuidos } \\
\text { en } 5 \text { dimensiones. } \\
\text { a) Inteligencia Interpersonal. } \\
\text { b) Inteligencia Intrapersonal. } \\
\text { c) Gestión del estrés. } \\
\text { d) Adaptación. } \\
\text { e) Estados de ánimos en general. } \\
\text { Enfocado a las áreas } \\
\text { administrativas (Trujillo y Rivas, } \\
\text { 2008). }\end{array}$ \\
\hline
\end{tabular}

Tabla 1 Modelos de instrumentos de inteligencia emocional. Fuente: Elaboración propia, basada en Fernández-Berrocal, Extremera, y Ramos, 2004; Trujillo y Rivas, 2005; Sánchez, Fernández-Berrocal, Montañés, y Latorre, 2008; Durán, 2013.

\section{Genos Inteligencia Emocional (IE)}

Genos EI fue diseñado con la idea de que mida el comportamiento promedio de los individuos en sus ambientes laborales (Palmer, Harmer, y Gignac, 2014), basado en que las áreas de recursos humanos evalúan el desempeño con indicadores típicos y no con máximos, sino como un rango típico de desempeño, es decir mide la frecuencia de los comportamientos del individuo en sus centros laborales en un momento en particular, donde se manifiesta la inteligencia emocional (G. Gignac, 2010) (G. E. Gignac, 2010).

La aplicación del instrumento tuvo lugar en diversos países alrededor del mundo, en América la muestra fue aplicada a 465 empleados con residencia en Estados Unidos, los cuales tenían una formación académica diversa, desde niveles de licenciatura hasta grados académicos de doctor.

Entre las bondades del inventario Genos de IE, está la medición de 7 factores entre los que encontramos, 5 factores basados en sí mismo y 2 basados en los otros.

1. Autoconciencia emocional.

2. Expresión emocional.

3. Razonamiento emocional.

\section{Autogestión emocional.}

5. Autocontrol emocional.

6. Manejo emocional de los demás.

7. Gestión emocional de los demás.

La inteligencia emocional busca equilibrar las relaciones entre las personas, fortalecer las relaciones interpersonales, superando los conflictos, dando paso a una empatía social en los grupos de trabajo (Nuñez, 2002). Para alcanzarlo se requiere de un entorno que propicie un clima de comunicación dirigida a la comprensión de las diversas situaciones que se presentan en la vida laboral, enfatizando el objetivo de la organización, es decir, para su razón de ser, orientando las reacciones emocionales para propiciar un ambiente de cooperación, claves para el éxito profesional (López-Fernández, 2015).

El Genos IE, resalta la existencia de emociones positivas y negativas, las cuales podemos ver como resultado de las primeras en la satisfacción, el entusiasmo, optimismo, la motivación y el compromiso, mientras que las segundas se manifiestan en la ansiedad, enojo, frustración y decepción (Palmer, Stough, Harmer, y Gignac, 2009). La versión concisa del Genos IE presenta niveles de confiabilidad para su aplicación para investigación, así como en ámbitos educativos.

\section{Metodología aplicada}

El instrumento tiene 31 ítems, escala tipo Likert de 5 puntos, que va de casi nunca a casi siempre, considerando la frecuencia con que el individuo demuestra el comportamiento descrito, su aplicación fue dirigida a alumnos de posgrados (maestrías y doctorados) de una universidad pública del sureste de México, el cual se aplicó en diversas Divisiones Académicas de la universidad, en un solo momento y en el lugar donde se encontraran los alumnos, es decir se aplicó tanto en aulas como en laboratorios, previo consentimiento de los alumnos y con el conocimiento del alcance de la investigación, obteniendo un total de 332 cuestionarios aplicados. Dicha investigación es de tipo cuantitativo, no experimental, transversal, basado en estudio factorial exploratorio en el programa estadístico SPSS. 
En el campo de las ciencias sociales es importante la confiabilidad y validez de un instrumento, la evaluación de la consistencia interna del instrumento fue mediante la confiabilidad Alfa de Cronbach (Quero, 2010).

\section{Resultados obtenidos}

El modelo de medición que se propone para determinar la "Inteligencia emocional" presenta un valor general del coeficiente Alpha de Cronbach de .93 que se considera confiable y comprueba su consistencia interna (Ho, 2015).

Un total de 332 alumnos encuestados, de los cuales 152 hombres y 180 mujeres, el $74 \%$ de maestría y $26 \%$ de doctorado, los resultados de confiablidad mediante Alfa de Cronbach para cada una de las dimensiones objeto de estudio se muestran en la tabla 2.

\begin{tabular}{|c|c|c|}
\hline \multicolumn{3}{|c|}{ Estadísticas de confiabilidad } \\
\hline Genos IE & $\begin{array}{l}\text { Alfa de } \\
\text { Cronbach }\end{array}$ & $\begin{array}{ll}\text { No. de } \\
\text { Items }\end{array}$ \\
\hline Autoconciencia emocional. & .774 & 4 \\
\hline Autocontrol emocional. & .813 & 4 \\
\hline Autogestión emocional. & .815 & 5 \\
\hline $\begin{array}{l}\text { Conciencia emocional de los } \\
\text { demás. }\end{array}$ & .832 & 4 \\
\hline Expresión emocional. & .802 & 5 \\
\hline Manejo emocional de los demás. & .872 & 4 \\
\hline Razonamiento emocional. & .847 & 5 \\
\hline
\end{tabular}

Tabla 2 Confiabilidad mediante alfa de cronbach para las dimensiones de Inteligencia Emocional

Los resultados del análisis factorial exploratorio realizado en SPSS, al instrumento de inteligencia emocional, fue realizado con el método de máxima verosimilitud con rotación de Oblimin directo, teniendo como resultado la medida de adecuación muestral del Kaiser-Meyer-Olkin adecuada KMO .927 , la prueba de esfericidad de Barlett fue significativa ( $p=.000$ ), por su parte, el valor de la varianza explicada fue de $53.89 \%$, explicado con ello un modelo de seis factores, contrastando con ello la teoría, la cual considera siete factores para el Genos IE (Palmer, et al., 2009). El análisis de correlación de las variables muestran una débil correlación entre las variables de autoconciencia emocional y autocontrol emocional, sin embargo la correlación es significativa al .05 , el resto de las variables presentan valores aceptables por arriba de .30 (Hernandez, Fernández, y Baptista, 2014) encontrando la más alta en la correlación entre la dimensiones de autogestión emocional y autocontrol emocional alcanzando un valor de .708

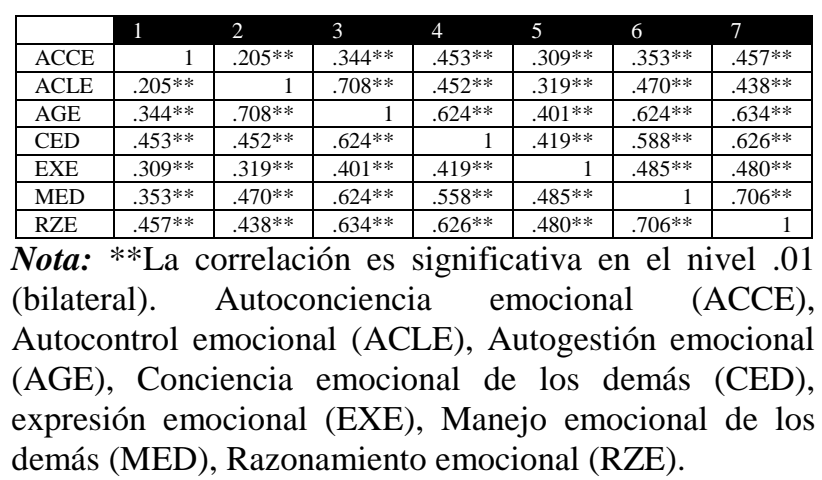

Tabla 3 Correlación de las variables del instrumento Genos IE

Los resultados de las cargas factoriales muestran que todos los ítems presentan un coeficiente de configuración sobre .30 que de acuerdo con Martínez, Pérez, León y Carmona (2018) es el valor mínimo sugerido para aceptar una carga factorial como adecuada.

La tabla 3 muestra los resultados de la composición de los seis factores obtenidos del análisis en el programa estadístico SPSS.

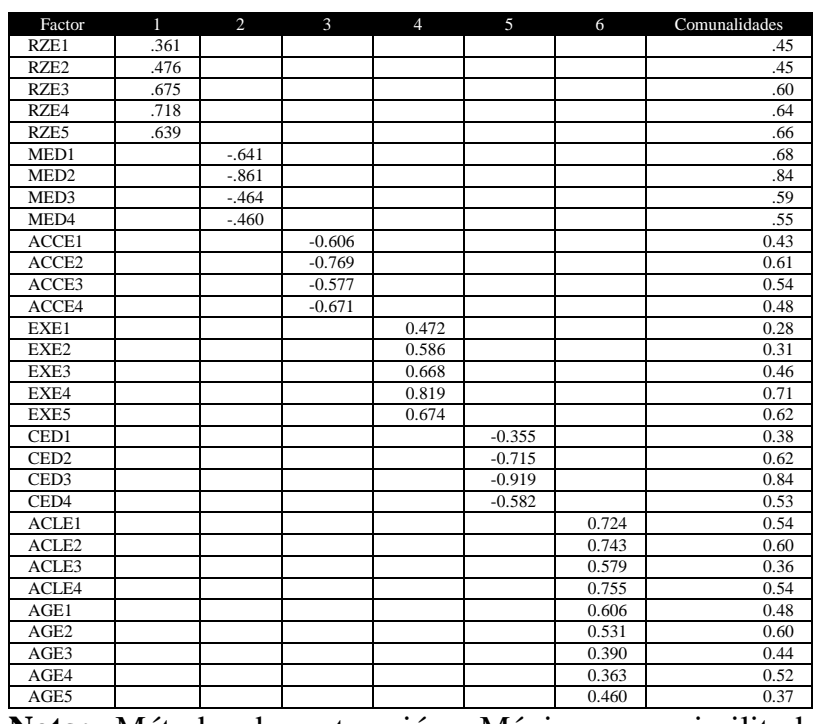

Nota: Método de extracción: Máxima verosimilitud, Método de rotación: Oblimin directo. $\mathrm{N}=332$

Tabla 4 Factores resultantes del análisis factorial exploratorio, de las dimensiones del Genos de Inteligencia Emocional

Los datos muestran la conservación de los ítems en las dimensiones de razonamiento emocional (RZE), manejo emocional de los demás (MED), autoconocimiento emocional (ACCE), expresión emocional (EXE), conciencia emocional (CED), fusionándose las dimensiones de autocontrol emocional (ACLE) y autogestión emocional (AGE) formándose con una nueva dimensión con nueve ítems la cual para objeto de esta investigación será nombrada como AUTOGESTIÓN EMOCIONAL, tomando como base los conceptos siguientes: 
Goleman (2002) considera entre los dominios de las competencias personales a la autogestión, agrupando en ellas a los conceptos de autocontrol emocional, la capacidad de adaptación, la iniciativa, el logro y el optimismo, mientras que Bisquerra y Pérez (2007) amplían el concepto de autogestión, agregándole concepto como la autoestima, capacidad de análisis crítico, así como identificar la necesidad de buscar ayuda o recursos, así como la resiliencia.

Esta estructura factorial difiere de lo propuesto por los autores considerando los siete factores, pese a este resultado, los factores logran conservar su capacidad de identificación de la inteligencia emocional manteniendo valores considerablemente positivos.

\section{Agradecimiento}

La presente investigación fue realizada gracias a los recursos recibidos por la estudiante mediante la beca conacyt.

\section{Conclusión}

Los resultados obtenidos confirman la confiabilidad del instrumento, apoyan a un modelo de seis factores, contradiciendo a los siete que mencionan los teóricos en su modelo, lo cual requiere mayor profundidad en los estudios posteriores, sin embargo, los resultados demuestran niveles de confiabilidad importantes en la toma de decisiones en lo referente a su uso con fines de reclutamiento y selección, ya que está diseñado para su aplicación en adultos y enfocado al ámbito laboral.

La evidencia encontrada favorece la utilización del Genos IE, con un Alfa de Cronbach de .93 se perfila como una herramienta que se suma a las existentes para la autoevaluación de la inteligencia emocional.

\section{Referencias}

Agreda, S. (2016). Nuevos retos en el reclutamiento y selección de personal: perspectivas organizacionales y divergencias éticas. Universidad Mariana, Boletín Informativo, 1(33), 66-74. Recuperado de http://www.umariana.edu.co/ojseditorial/index.php/BoletinInformativoCEI/artic le/view/924/849
Bisquerra, R., y Pérez, N. (2007). Las competencias emocionales. Educación XX1, $10(0)$, $61-82$.

https://doi.org/10.5944/educxx1.1.10.297

Calderón, M., González, G., Salazar, P., y Washburn, S. (2012). Aprendiendo sobre emociones. Manual de Educación Emocional.

De José, M., Danvila, I., Martínez-López, F., y Sastre, M. A. (2013). Jerarquización de competencias emocionales a través del modelo de la pirámide invertida. Revista Venezolana de Gerencia, 18(61), 43-61.

Duque, J., García, M., y Hurtado, A. (2017). Influencia de la inteligencia emocional sobre las competencias laborales: un estudio empírico con empleados del nivel administrativo. Estudios Gerenciales (2017), https://doi.org/10.1016/j.estger.2017.06.005

Duque, José, García, M., y Hurtado, A. (2017). Influencia de la inteligencia emocional sobre las competencias laborales: un estudio empírico con empleados del nivel administrativo. Estudios Gerenciales, 33(36), 250-260. https://doi.org/10.1016/j.estger.2017.06.005

Durán, P. (2013). Adaptación y Validación del Cuestionario de inteligencia emocional TMMS48 de Salovey y Mayer, en estudiantes de pregrado de la Facultad de educacion y Humanidades y de la Facultad de Ciencias de la Salud y Alimentos de la Universidad del Bío Bío de la Ci. Escuela de Psicologia, Tesis.

Extremera, N., y Fernández, P. (2005). La Inteligencia Emocional y la educación de las emociones. Revista Interuniversitaria de Formacion Del Profesorado, 19(3), 63-93. Retrieved from http://www.redalyc.org/articulo.oa?id=2741192 7005\%0ACómo

Fernández-Berrocal, P, Extremera, N., y Ramos, N. (2004). Tmms-24. Revista Latinoamericana de Psicología, 36(2), 209-228. https://doi.org/10.1157/13061586

Fernández-Berrocal, Pablo, Extremera, N., y Durán, A. (2003). Inteligencia emocional y burnout en profesores. Encuentros En Psicología Social, (September 2017), 260-265. 
Gignac, G. (2010). Seven-factor model of emotional intelligence as measured by Genos EI: A confirmatory factor analytic investigation based on self-and rater-report data. European Journal of Psychological Assessment, 26(4), 309-316. https://doi.org/10.1027/1015$5759 / \mathrm{a} 000041$

Gignac, G. E. (2010). On a Nomenclature for Emotional Intelligence Research. Industrial and Organizational Psychology, 3(2), 131-135. https://doi.org/10.1111/j.1754-

9434.2010.01212.x

Gil, J. (2007). La Evaluación de Competencias Laborales. Educación XX1, 10, 83-106. Recuperado de http://www.redalyc.org/articulo.oa?id=7060100 6

Goleman, D. (2002). El Líder resonante crea más. Resumen National Business School, 5. Recuperado de www.nbs.com.gt

Goleman, D. (2015). Cerebro y la inteligencia emocional. Retrieved from https://books.google.es/books?hl=es\&lr=\&id=0 _kVCgAAQBAJ\&oi=fnd\&pg=PT8\&dq=+inteli gencia+emocional\&ots=Obf1NAkW68\&sig=y ZP0QTI7ukR371TsO_GV4fxZOss

Hernández-Vargas, C., y Dickinson-Bannack, M. E. (2014). Importancia de la inteligencia emocional en Medicina. Investigación En Educación Médica, 3(11), 155-160. https://doi.org/10.1016/S2007-5057(14)727425

Hernandez, R., Fernández, C., y Baptista, M. (2014). Metodología de la investigación. (6a edición). McGraw Hill.

Ho, R. (2015). Manual de análisis de datos univariados y multivariado y su interpretacion con SPSS.

García-Izquierdo, A., García-Izquierdo, M., y Ramos-Villagrasa, P. J. (2007). Aportaciones de la inteligencia emocional y la autoeficacia: Aplicaciones para la selección de personal, 23(2), 231-239. Recuperado de http://digitum.um.es/jspui/handle/10201/8133
López-Fernández, C. (2015). Inteligencia emocional y relaciones interpersonales en los estudiantes de enfermería. Educacion Medica, 16(1), 83-92. https://doi.org/10.1016/j.edumed.2015.04.002

Lozano, J., y González, C. (2015). Un análisis del estado del arte de la inteligencia organizacional (IO), con sus modelos y herramientas de diagnostico. Revistas de Ciencas Estrategias, 23(33), 41-52. https://doi.org/dx.doi.org/10.18566/rces.v23n33 $\mathrm{a} 03$

Martinez, R., Pérez, C., León, H. L., y Carmona, L. (2018). Estructura factorial y consistencia interna de cuestionario de evaluación de docentes clínicos en el internado de fonoaudiología. Revista Cubana de Educación Médica Superior., 32(2), 1-10. Recuperado de http://scielo.sld.cu/pdf/ems/v32n2/a06_1264.pd $\mathrm{f}$

Newman, M., Purse, J., Smith, K., y Broderick, J. (2017). Assessing Emotional Intelligence in Leaders and Organisations: Reliability and Validity of the Emotional Capital Report ( ECR ). Australasian Journal of Organisational Psychology, $8, \quad 1-15$. https://doi.org/10.1017/orp.2015.5

Nuñez, I. (2002). Inteligencia emocional en las entidades de información. ¿ Cómo encauzar los afectos en bien de la organización? ACIMED, 10(1), 77-82.

OCDE, U. (2005). La definición y selección de competenicias claves. DeSeCo. OCDE. Unesco. Recuperado de http://www.deseco.admin.ch/bfs/deseco/en/inde $\mathrm{x} / 03 / 02 . \mathrm{html}$

Palmer, B. R., Stough, C., Harmer, C., y Gignac, G. E. (2014). Emotional Intelligence Inventory Concise. Genos.

Palmer, B., Stough, C., Harmer, R., y Gignac, G. (2009). The Genos emotional intelligence inventory: a measure designed specifically for workplace applications. In assessing Emotional Intelligence. (pp. 103-117). https://doi.org/10.1007/978-0-387-88370-0 
Pino del, R., y Aguilar, M. (2013). La inteligencia emocional como una herramienta de la gestión educativa para el liderazgo estudiantil. Cuadernos de Administración, Universidad Del Valle, 29(50), 132-141.

Quero, M. (2010). Confiabilidad y coeficiente de Alpha de Cronbach. Revista de Estudios Interdisciplinarios de Ciencias Sociales, Vol. 12(2), 248-252.

Sánchez, M., Fernández-Berrocal, P., Montañés, J., y Latorre, J. (2008). ¿Es la inteligencia emocional una cuestión de género? Socialización de las competen cias emocionales en hombres y mujeres y sus implicaciones. Investigacionpsicopedagogicaorg, 6(2), 455474. Recuperado de http://www.investigacionpsicopedagogica.org/revista/articulos/15/espann ol/Art_15_253.pdf

Sánchez, T. M., Montañés, J., Latorre, J. M., \& Fernández-berrocal, P. (2008). ¿Es la inteligencia emocional una cuestión de género? Socialización de las competencias emocionales en hombres y mujeres y sus implicaciones. Electronic Journal of Research in Educational Psychology, 6(15), 455-474. Recuperado de from

http://www.redalyc.org/articulo.oa?id=2931219 24011

Serrano, G. (2017). Competencias directivas y virtudes: un camino a la excelencia. Estudios Gerenciales, 33(143), 208-216. https://doi.org/10.1016/j.estger.2017.03.004

Trujillo, M. M., y Rivas, L. A. (2008). Emotional intelligence model for Directors of Research centers in Mexico. Estudios Gerencias, 24(106), 13-30.

Trujillo, M., y Rivas, L. A. (2005). Origen, evolución y modelos de inteligencia emocional. INNOVAR, (1), 9-24.

Zárate, R. A., y Matviuk, S. (2012). Inteligencia Emocional y prácticas de liderazgo en las organizaciones colombianas. Cuadernos de Administración, Universidad Del Valle, 28(47), 91-104. 